\title{
Microstructural changes in thickened corpus callosum in children: contribution of magnetic resonance diffusion tensor imaging
}

\author{
Laura Merlini • Mehrak Anooshiravani • \\ Aikaterini Kanavaki • Sylviane Hanquinet
}

Received: 18 March 2014 /Revised: 7 October 2014 / Accepted: 12 November 2014 /Published online: 3 December 2014

(C) Springer-Verlag Berlin Heidelberg 2014

\begin{abstract}
Background Thickened corpus callosum is a rare finding and its pathophysiology is not well known. An anomalous supracallosal bundle has been depicted by fiber tracking in some cases but no diffusion tensor imaging metrics of thickened corpus callosum have been reported.

Objective To use diffusion tensor imaging (DTI) in cases of thickened corpus callosum to help in understanding its clinical significance.

Materials and methods During a 7-year period five children (ages 6 months to 15 years) with thickened corpus callosum were studied. We determined DTI metrics of fractional anisotropy (FA), mean diffusivity, and axial $(\lambda 1)$ and radial $(\lambda 2, \lambda 3)$ diffusivity and performed 3-D fiber tracking reconstruction of the thickened corpus callosum. We compared our results with data from the literature and 24 age-matched controls.

Results Brain abnormalities were seen in all cases. All children had at least three measurements of corpus callosum thickness above the 97th percentile according to age. In all children 3-D fiber tracking showed an anomalous supracallosal bundle and statistically significant decrease in FA $(P=0.003)$ and $\lambda 1(P=0.001)$ of the corpus callosum compared with controls, but no significant difference in mean diffusivity and radial diffusivity.

Conclusion Thickened corpus callosum was associated with abnormal bundles, suggesting underlying axonal guidance abnormality. DTI metrics suggested abnormal fiber compactness and density, which may be associated with alterations in cognition.
\end{abstract}

L. Merlini $(\bowtie) \cdot$ M. Anooshiravani $\cdot$ A. Kanavaki $\cdot$ S. Hanquinet Pediatric Radiology Unit, University of Geneva Children's Hospital, Rue Willy-Donzé 6, Geneva 1205, Switzerland

e-mail: laura.merlini@hcuge.ch
Keywords Corpus callosum · Diffusion tensor imaging · Fiber tracking · Supracallosal bundle $\cdot$ Children . Magnetic resonance imaging

\section{Introduction}

The finding of a thickened corpus callosum (Fig. 1) is rarely reported, most likely because of its variability in size during development [1]. Nevertheless a recent paper provides reliable biometric data of the corpus callosum according to age [2].

The pathophysiology of thickened corpus callosum is not well known because it is in some cases an isolated finding [3, 4]; however it is frequently associated with other brain malformations [3,5-10]. It can be a part of syndromic conditions such as Cohen syndrome [11], Williams syndrome [12], and neurofibromatosis type 1 [13]. Non-neurological abnormalities reported in association with thickened corpus callosum are hypertrichosis and hyperkeratosis [14] and Wilms tumor [15].

Because of its importance in cognition the corpus callosum is one of the most common white matter structures studied with diffusion tensor imaging (DTI). Abnormal DTI metrics are considered to be a sign of structural abnormalities, which can cause alterations in cognition [16]. However no DTI metrics of thickened corpus callosum have been reported. Two recent papers reported that DTI and fiber tract reconstruction can show anomalous longitudinal supracallosal fibers separated from the cingulum in congenital cases of thickened corpus callosum $[3,17]$. One case was also analyzed histologically [17]. Using five cases of thickened corpus callosum, we describe clinical presentation, MR findings, fiber tracking reconstructions and DTI metrics to help in understanding the clinical significance of this brain anomaly. 


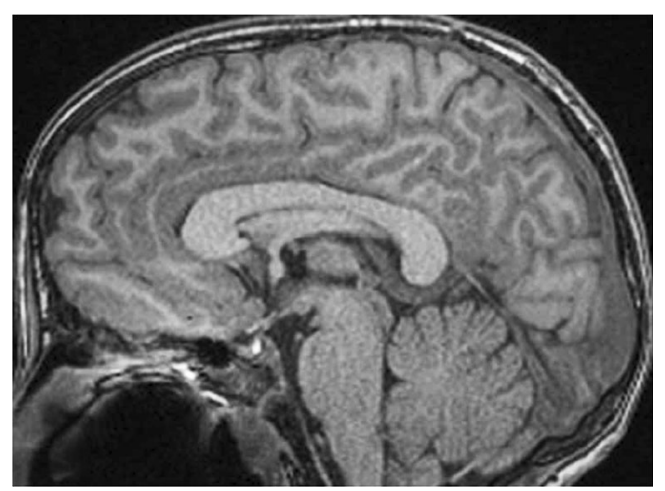

Fig. 1 Hemimegalencephaly and polymicrogyria in a 13-year-old boy (patient 2). Mid-sagittal T1-weighted MR sequence illustrates global thickening of the corpus callosum

\section{Materials and methods}

We reviewed medical records of five children with thickened corpus callosum. The cases, spanning 2002 to 2013, were retrieved from our department's PACS search engine using the keywords "thick and/or mega corpus callosum." The children were included when at least three measurements of the thickness of the corpus callosum were above the 97th percentile according to age [2]. Our institutional review board approved this retrospective study (CER 12-102R).

MR studies were performed using a 1.5T Avanto MRI scanner (Siemens, Erlangen, Germany) and included T2weighted and 3-D T1-weighted and DTI sequences. The DTI sequences were acquired with the following parameters: echo time $(\mathrm{TE})=92 \mathrm{~ms}$, repetition time $=9,000 \mathrm{~ms}$, integrated parallel acquisition technique $=2, \mathrm{Nex}=1$, field of view $=$ 230 , matrix $=128 \times 128$, slice thickness $=2 \mathrm{~mm}$, resulting in a voxel size of $2 \times 2 \times 2 \mathrm{~mm}$, b values $=0$ and $1,000 \mathrm{~s} / \mathrm{mm} 2$, and 30 gradient directions. Diffusion weighting implementation (using a Stejskal-Tanner diffusion scheme), fractional anisotropy (FA), apparent diffusion coefficient maps and tensor calculation were automatically performed by the scanner.

Corpus callosum measurements were taken by two senior radiologists (L.M. and S.H., with 15 and 25 years of experience, respectively) in pediatric radiology. Measurements were from a midsagittal plane 3-D T1weighted spin-echo image that clearly demonstrated the pituitary infundibulum, the cerebral aqueduct, the pineal gland, and the corpus callosum in a single image with the Osirix DICOM viewer (free open source).

The two radiologists first analyzed several anonymous cases together to agree on the method. Then each radiologist, blinded to patient data, took the measurements individually in all patients and controls. In discordant cases, they reached a consensus.

Measurements were based on a previous study on MR biometric data of the corpus callosum [2]: the distance between the anterior part of the genu and the posterior aspect of the splenium, the curvilinear distance between the rostrum and the splenium at mid-thickness of the corpus callosum, and the thickness of the corpus callosum at the level of the genu, body, isthmus and splenium. Our inclusion criteria were at least three measurements of the corpus callosum above the 97th percentile according to age [2].

We also calculated the corpus callosum area with the Osirix brush tool, performed on the same plane by manual tracing according to the method described by Mitchell et al. [18]. The mean area of the corpus callosum and standard deviation (SD) of the repeated measurements were calculated. These values were compared with mean values calculated in the same manner in 24 age-matched healthy controls imaged for screening purposes (i.e. familiar vascular malformations) or extra-axial soft-tissue tumors. We separated comparative analysis for the 6-month-old patient (patient 1) with four age-matched controls, and four patients older than 5 years with 20 age-matched controls.

Fiber tracking was performed with TrackVis software using the fiber assignment by a continuous tracking method [19]. The samples per voxel length were $1 \mathrm{~mm}$, with a step length of $1 \mathrm{~mm}$, a curvature threshold of 0.2 , an FA threshold of 0.2 , and an angle threshold of 35.0. For DTI measurements, we performed a separate analysis for infants (one patient and four controls) because the myelin immaturity accounts for different DTI metrics during the first year of life.

The same region of interest used for calculating the area was also used for fiber tracking reconstruction and to retrieve DTI metrics. FA values were measured as scalar values ranging $0-1$. Mean diffusivity, axial diffusivity $(\lambda 1)$, radial diffusivity $(\lambda 2, \lambda 3)$, mean values and standard deviation were measured as $\mathrm{mm}^{2} / \mathrm{s} \times 10^{-3}$.

We compared DTI metrics with values calculated in the same manner as in the control group. Comparisons of FA, mean diffusivity, $\lambda 1, \lambda 2$ and $\lambda 3$ values between the four older patients and 20 controls were assessed with the WilcoxonMann-Whitney test. Statistical significance was assessed at the 0.05 level for all analyses. For the patient younger than 1 year (patient 1) and the four age-matched controls we did not perform statistical analysis and only provide a description of DTI metrics data.

\section{Results}

We included three girls and two boys with thickened corpus callosum. Patient 1 was 6 months old; the others were older than 5 years (5-13 years). Controls for the younger age group were 2 girls and 2 boys (age range 5-7 months), and for the older group there were 10 girls and 10 boys (age range 5.4 15.3 years).

Table 1 summarizes age, clinical presentation, associated brain abnormalities and corpus callosum measurements of the 
five children in the study. All of these children had cognitive impairment, which was severe in patients 3 and 5, mild in patients 2 and 4, and was not yet tested in patient 1 . Two patients (patients 1 and 2) had pachy- and/or polymicrogyria; one of these children had associated hemimegalencephaly (patient 2). Two patients (patients 4 and 5) were diagnosed with Joubert syndrome and had classic signs of this pathology such as ataxia and oculomotor apraxia. Both presented the molar tooth appearance of the hind-brain. The last patient (patient 3) had anomalous bilateral hippocampi.

The mean value of corpus callosum area was $10,186 \pm$ $1,542 \mathrm{~cm}^{2}$ in older patients ( $>5$ years), which was significantly larger $(P<0.001)$ than in controls, in whom it was $5,400 \pm$ $0.833 \mathrm{~cm}^{2}$. For our one younger patient $(<1$ year) the corpus callosum area was $6,100 \mathrm{~cm}^{2}$ while the mean for age-matched controls was $2,490 \pm 0.481 \mathrm{~cm}^{2}$. For all children the corpus callosum area was more than two standard deviations above the mean for age-matched controls. In all children at least three of the measurements of corpus callosum thickness were above the 97th percentile (including criteria) according to age as reported by Garel et al. [2]. In patient 1, a 6-month-old girl, the splenium was thinner than normal $(3.8 \mathrm{~mm})$, less than the 3rd percentile $(4.3 \mathrm{~mm})$ in the paper of reference [2]. However, all other measurements were above the 97th percentile according to age (Table 1).

In all children fiber tracking reconstructions showed a pathological bundle on top of the corpus callosum with no relation to the cingular gyrus (Fig. 2). The anomalous bundle was represented in green (Fig. 3), indicating anteroposterior orientation, and was also easily identified between the paired cingula on color maps. The size of the pathological bundle was different in each child, depending on the quantity of fibers, which were thinner and inhomogeneous in patient 1 (Fig. 1) and thicker in other patients (Fig. 2).

Table 2 illustrates the statistical comparison of DTI metrics among the four children and 20 control subjects older than 1 year. There was a significant difference in FA $(P=0.003)$ and axial diffusivity $(P<0.001)$. Both were lower in our patients than in normal controls. For other measurements, the difference was not significant.

Table 3 shows the same metrics in the only patient $<1$ year of age (patient 1) and four age-matched controls: FA and axial diffusivity $(\lambda 1)$ were clearly lower in this child. Furthermore, $\lambda 2$ was slightly higher and there were no differences in mean diffusivity and $\lambda 3$ measurements.

\section{Discussion}

Thickened corpus callosum was identified in all five study patients, according to segmental measurements performed and compared to recently published biometric data as a function of 

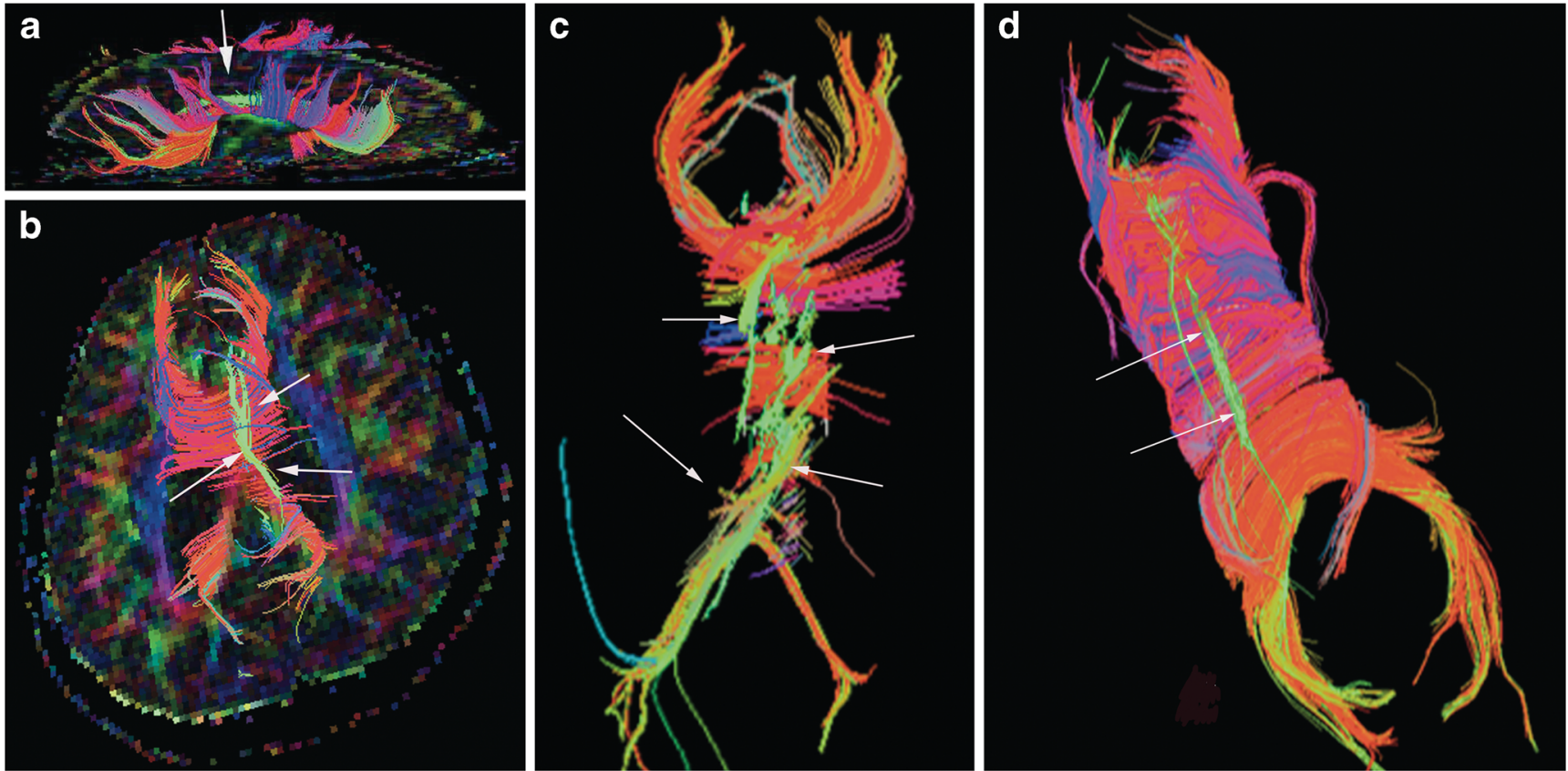

Fig. 2 Three-dimensional fiber tracking reconstruction of the thickened corpus callosum shows a different aspect of the anomalous fiber bundle on top of the corpus callosum in various children. a, b Imaging in a 5year-old boy (patient 3) shows the anomalous bundle as thick and continuous (arrows). c Imaging in a 6-month-old girl (patient 1) shows

that the fibers of the corpus callosum are scarce on the splenium and the anomalous bundle is interrupted and deviated on the right side (arrows). d Imaging in a 7-year-old girl (patient 5) shows that the anomalous bundle is thinner than in the other patients (arrows)

age [2]. Moreover, the calculated corpus callosum area was higher than in the age-matched controls.

During fetal life, the surface and the length of the corpus callosum gradually increases after the 20th gestational week to

reach a maximum at the 28th gestational week [20]. From the 28th gestational week onward the number of axons in the corpus callosum drastically declines until it reaches a minimum at the 35 th gestational week. After the 35 th gestational

Fig. 3 Anomalous midline fibers in a 10-year-old girl (patient 4) with thickened corpus callosum. Sagittal (a), axial (b) and coronal (c) fractional anisotropy color maps show the anomalous midline fibers (white arrows), clearly separable from the paired cingulum (red arrowheads)
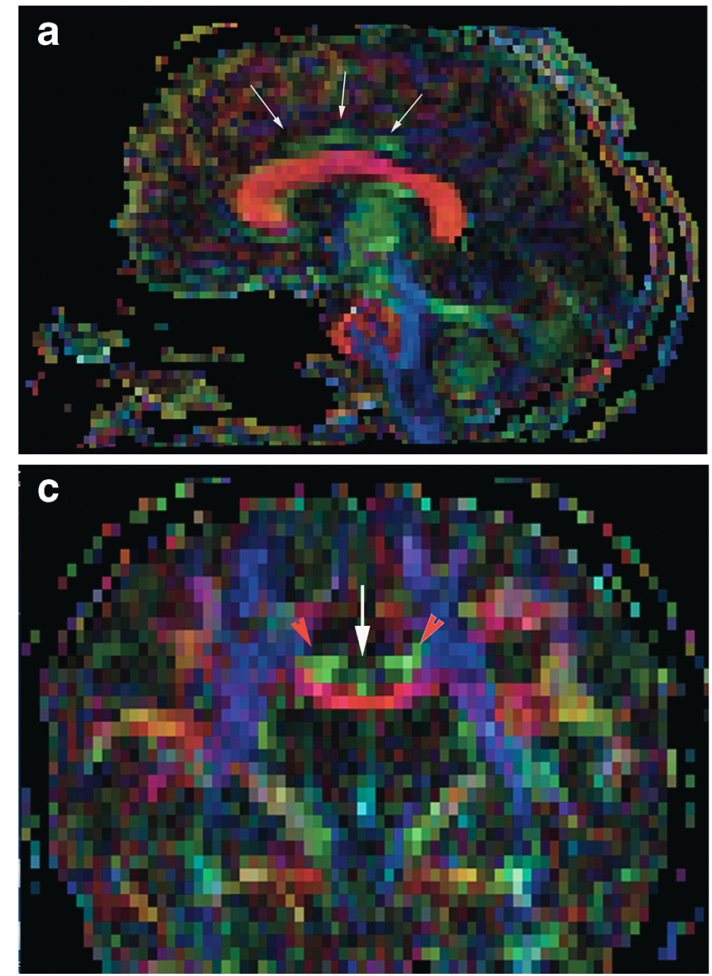

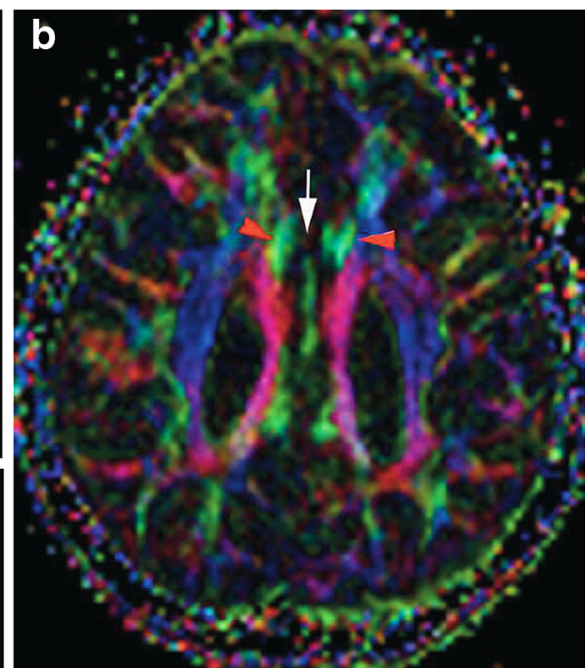


Table 2 Older children. Mean \pm standard deviation values of diffusion tensor imaging (DTI) measurements in children older than 1 year and agematched controls

\begin{tabular}{lccl}
\hline & Patients $n=4$ & Controls $n=20$ & $P$-value \\
\hline MD & $0.850 \pm 11$ & $0.900 \pm 0.06$ & 0.360 \\
$\mathrm{FA}^{\mathrm{b}}$ & $0.58 \pm 0.79$ & $0.69 \pm 0.28$ & $0.003^{\mathrm{d}}$ \\
$\lambda 1^{\mathrm{c}}$ & $0.150 \pm 0.016$ & $0.189 \pm 0.013$ & $0.001^{\mathrm{d}}$ \\
$\lambda 2^{\mathrm{c}}$ & $0.065 \pm 0.013$ & $0.064 \pm 0.005$ & 0.897 \\
$\lambda 3^{\mathrm{c}}$ & $0.045 \pm 0.011$ & $0.034 \pm 0.004$ & 0.256 \\
\hline
\end{tabular}

$A D C$ apparent diffusion coefficient, $\lambda l$ axial diffusivity, $\lambda 2$ and $\lambda 3$ radial diffusivity, $F A$ fractional anisotropy, $M D$ mean diffusivity

${ }^{\text {a }}$ Wilcoxon-Mann-Whitney test

${ }^{\mathrm{b}}$ FA measurements are a scalar value ranging $0-1$

${ }^{c} \lambda 1, \lambda 2$ and $\lambda 3$ mean values and standard deviations are measured in $\mathrm{mm}^{2} / \mathrm{s} \times 10^{-3}$

${ }^{\mathrm{d}}$ Statistically significant

week it again starts to increase until reaching the maximum value during late adolescence [21]. Thus one can speculate that in our patients a pathological event occurred after the 28th gestational week that prevented the elimination of crossing axons and resulted in a thickened corpus callosum. Associated brain malformations, such as hippocampal abnormalities [22, 23], megalencephaly from a default in cellular proliferation, and micro/pachygyria from a default in cellular migration $[5$, 10] could have occurred at the same fetal period. It must be noted that there is a relationship between the corpus callosum and hippocampi during fetal development [23, 24].

Decline in the surface area of the corpus callosum from the 28 th to the 35 th gestational weeks is thought to be secondary to microstructural changes, such as a decrease in the number of axonal elements or an increase in density, as reported in animal models [25-27]. Our study confirms microstructural changes in the fibers in a thickened corpus callosum, showing a significant decrease in FA $(P=0.002)$ and axial white matter diffusion $(\lambda 1)(P<0.001)$ across the entire corpus callosum in

Table 3 Younger children. Diffusion tensor imaging (DTI) measurements in the only patient younger than 1 year, in comparison to four similar-age controls. Values given in mean plus or minus standard deviation

\begin{tabular}{llc}
\hline & Patient 1 (6 months) $n=1$ & Controls $n=4$ \\
\hline MD & 0.122 & $0.132 \pm 0.6 .5$ \\
$\mathrm{FA}^{\mathrm{a}}$ & 0.32 & $0.48 \pm 0.033$ \\
$\lambda 1^{\mathrm{b}}$ (axial) & 0.168 & $0.245 \pm 0.004$ \\
$\lambda 2^{\mathrm{b}}$ (radial) & 0.111 & $0.90 \pm 0.7$ \\
$\lambda 3^{\mathrm{b}}$ (radial) & 0.60 & $0.64 \pm 0.0008$ \\
\hline
\end{tabular}

$\lambda 1$ axial diffusivity, $\lambda 2$ and $\lambda 3$ radial diffusivity, $F A$ fractional anisotropy, $M D$ mean diffusivity

${ }^{\text {a }}$ FA measurements are a scalar value ranging $0-1$

${ }^{b} \mathrm{ADC}, \lambda 1, \lambda 2$ and $\lambda 3$ mean values and standard deviations are measured on $\mathrm{mm}^{2} / \mathrm{s} \times 10^{-3}$ children with thickened corpus callosum (Table 2). Radial diffusivity being normal, it seems more likely that there is abnormal fiber compactness and density rather than abnormal myelination $[28,29]$.

The interesting finding in our study is the presence of an aberrant longitudinal supracallosal fiber bundle, separated from the cingulum in all five children with thickened corpus callosum. This particular bundle has been described in association with thickened corpus callosum [3, 17] but also in patients with no thickened corpus callosum [30]. Transitory corpus callosum axons have been described during development [27]. In our patients, they could have persisted pathologically. Some authors consider this bundle to be an aberrant cingulum or commissural axons that have gone astray during axonal migration [30].

The hypothesis of an underlying axonal guidance abnormality could be supported by the observation that two of our patients (patients 4 and 5) had Joubert syndrome-related disorders, a ciliopathy with abnormal decussation pathways [31]. This association has not been previously reported.

Our study has some limitations. We had a small and heterogeneous group of patients because of the rarity of the thickened corpus callosum pathology. We did not use a segmental approach to analyzing the genu, body and splenium separately. We limited our investigation to the corpus callosum; microstructural changes we found may be more diffuse and widespread in other bundles and tracks, but this was beyond the scope of our study. Moreover, we did not use high-angular-diffusion imaging with multiple $b$ values, which would have been preferable for purposes of determining connectivity. This technique is not feasible in a clinically acceptable acquisition time for children. It also would have been more appropriate for our purposes to have used neurite orientation dispersion and density imaging (NODDI), an interesting MR technique for estimating the microstructural complexity of dendrites and axons [32] that is feasible in children [33]. However because our study was retrospective, it was difficult to repeat MR studies using this relatively recent technique. Finally, we do not have any anatomical-pathological proof of the real existence of the aberrant fiber bundle in the corpus callosum.

\section{Conclusion}

Our study suggests that thickened corpus callosum is not just a morphological abnormality but implies microstructural changes with abnormal fiber compactness and density. These abnormalities of the DTI parameters of the corpus callosum may help to explain the neurocognitive difficulties in these children. Moreover, the finding of an abnormal supracallosal fiber bundle suggests an underlying axonal guidance abnormality. 
Acknowledgments We are grateful to Antoine Ponçet for his assistance in statistical analysis and to Seema Toso for her editorial assistance.

Conflicts of interest None

\section{References}

1. Giedd JN, Blumenthal J, Jeffries NO et al (1999) Development of the human corpus callosum during childhood and adolescence: a longitudinal MRI study. Prog Neuropsychopharmacol Biol Psychiatry 23: 571-588

2. Garel C, Cont I, Alberti C et al (2011) Biometry of the corpus callosum in children: MR imaging reference data. AJNR Am J Neuroradiol 32:1436-1443

3. Rollins NK (2013) Diffusion imaging of the congenitally thickened corpus callosum. AJNR Am J Neuroradiol 34:660-665

4. Lerman-Sagie T, Ben-Sira L, Achiron R et al (2009) Thick fetal corpus callosum: an ominous sign? Ultrasound Obstet Gynecol 34: 55-61

5. Bindu PS, Taly AB, Sinha S et al (2010) Mega-corpus callosum, polymicrogyria, and psychomotor retardation syndrome. Pediatr Neurol 42:129-132

6. Göhlich-Ratmann G, Baethmann M, Lorenz P et al (1998) Megalencephaly, mega corpus callosum, and complete lack of motor development: a previously undescribed syndrome. Am J Med Genet 79:161-167

7. Hengst M, Tücke J, Zerres K et al (2010) Megalencephaly, mega corpus callosum, and complete lack of motor development: delineation of a rare syndrome. Am J Med Genet 152A: 2360-2364

8. Dagli AI, Stalker HJ, Williams CA (2008) A patient with the syndrome of megalencephaly, mega corpus callosum and complete lack of motor development. Am J Med Genet 146A:204-207

9. Rypens F, Sonigo P, Aubry MC et al (1996) Prenatal MR diagnosis of a thick corpus callosum. AJNR Am J Neuroradiol 17:1918-1920

10. Pierson TM, Zimmerman RA, Tennekoon GI et al (2008) Mega-corpus callosum, polymicrogyria, and psychomotor retardation: confirmation of a syndromic entity. Neuropediatrics 39:123-127

11. Kivitie-Kallio SH, Autti TH, Salonen OH et al (1998) MRI of the brain in the Cohen syndrome: a relatively large corpus callosum in patients with mental retardation and microcephaly. Neuropediatrics 29:298-301

12. Sampaio A, Bouix S, Sousa N et al (2013) Morphometry of corpus callosum in Williams syndrome: shape as an index of neural development. Brain Struct Funct 218:711-720

13. Dubovsky EC, Booth TN, Vezina G et al (2001) MR imaging of the corpus callosum in pediatric patients with neurofibromatosis type 1. AJNR Am J Neuroradiol 22:190-195

14. Pöyhönen MH, Peippo MM, Valanne LK et al (2004) Hypertrichosis, hyperkeratosis, abnormal corpus callosum, mental retardation and dysmorphic features in three unrelated females. Clin Dysmorphol 13:85-90
15. Cavicchioni O, Gomes DM, Leroy B et al (2005) Prenatal diagnosis of de novo (7;19)(q11.2;q13.3) translocation associated with a thick corpus callosum and Wilms tumor of the kidneys. Prenat Diagn 25: 876-878

16. Filippi CG, Watts R, Duy LA et al (2013) Diffusion-tensor imaging derived metrics of the corpus callosum in children with neurofibromatosis type I. AJR Am J Roentgenol 200:44-49

17. Koob M, Weingertner AS, Gasser B et al (2012) Thick corpus callosum: a clue to the diagnosis of fetal septopreoptic holoprosencephaly? Pediatr Radiol 42:886-890

18. Mitchell TN, Free SL, Merschhemke M et al (2003) Reliable callosal measurement: population normative data confirm sex-related differences. AJNR Am J Neuroradiol 24:410-418

19. Mori S, Crain BJ, Chacko VP et al (1999) Three-dimensional tracking of axonal projections in the brain by magnetic resonance imaging. Ann Neurol 45:265-269

20. Kadhim HJ, Bhide PG, Frost DO (1993) Transient axonal branching in the developing corpus callosum. Cereb Cortex 3:551-566

21. LaMantia AS, Rakic P (1994) Axon overproduction and elimination in the anterior commissure of the developing rhesus monkey. J Comp Neurol 340:328-336

22. Göçmen R, Oğuz KK (2008) Mega corpus callosum and caudate nuclei with bilateral hippocampal malformation. Diagn Interv Radiol 14:69-71

23. Raybaud C (2010) The corpus callosum, the other great forebrain commissures, and the septum pellucidum: anatomy, development, and malformation. Neuroradiology 52:447-477

24. Di Ieva A, Fathalla H, Cusimano MD et al (2014) The indusium griseum and the longitudinal striae of the corpus callosum. Cortex. doi:10.1016/j.cortex.2014.06.016 [Epub ahead of print]

25. Karol EA, Pandya DN (1971) The distribution of the corpus callosum in the rhesus monkey. Brain 94:471-486

26. LaMantia AS, Rakic P (1990) Axon overproduction and elimination in the corpus callosum of the developing rhesus monkey. J Neurosci 10:2156-2175

27. Elberger AJ (1994) Transitory corpus callosum axons projecting throughout developing rat visual cortex revealed by Dil. Cereb Cortex 4:279-299

28. Song SK, Sun SW, Ramsbottom MJ (2002) Dysmyelination revealed through MRI as increased radial (but unchanged axial) diffusion of water. Neuroimage 17:1429-1436

29. Tovar-Moll F, Moll J, de Oliveira-Souza R et al (2007) Neuroplasticity in human callosal dysgenesis: a diffusion tensor imaging study. Cereb Cortex 17:531-541

30. Hori A, Stan AC (2004) Supracallosal longitudinal fiber bundle: heterotopic cingulum, dorsal fornix or Probst bundle? Neuropathology 24:56-59

31. Poretti A, Meoded A, Rossi A et al (2013) Diffusion tensor imaging and fiber tractography in brain malformations. Pediatr Radiol 43:28-54

32. Zhang H, Schneider T, Wheeler-Kingshott CA et al (2012) NODDI: practical in vivo neurite orientation dispersion and density imaging of the human brain. Neuroimage 61:1000-1016

33. Kunz N, Zhang H, Vasung L et al (2014) Assessing white matter microstructure of the newborn with multi-shell diffusion MRI and biophysical compartment models. Neuroimage 96:288-299 